\title{
Feedback Optimal Control for Inverted Pendulum Problem by Using the Generating Function Technique
}

\author{
Hany R. Dwidar \\ Astronomy, Meteorology and Space Science Dept. \\ Faculty of Science - Cairo University \\ Giza - EGYPT 12613
}

\begin{abstract}
In this paper, a model is described for a system consisting of an inverted pendulum attached to a cart. We design for this model a feedback optimal control based on Linear Quadratic regulator, LQR by using the generating Function technique. This design with hard and soft constraints will help the pendulum to stabilize in the upright position. A solution of the continuous low-thrust optimal control problem based on LQR method is implemented. An example applied to this control design for a hard constraint boundary condition.
\end{abstract}

Keywords-Inverted pendulum; Feedback control; Stability analysis

\section{INTRODUCTION}

The traditional problem for the field of control systems is the inverted pendulum system see e.g. [1] , [3] , [5] , [6] and [9]. The system is consist of an inverted pendulum exposed to a torque and attached to a cart which equipped with a motor that drives it along a friction horizontal track. Both the torque and the force produced from the motor of the cart are the feedback-control forces. there are two equilibrium points for the inverted pendulum system, one of them is that when the pendulum is pointing downwards which is stable, the other one is at the upwards position which is unstable. The stable equilibrium requires no control input to be achieved and, thus, is uninteresting from a control perspective. The unstable equilibrium corresponds to a state in which the pendulum points strictly upwards and, thus, requires a control force to maintain this position.

In literature, the feedback control of inverted pendulum control system is made by linearizing the dynamics about the nominal trajectory and by applying the classic control theory to such linear dynamical system. The approach of optimal feedback control using the generating function [8] is very efficient when used to solve the control problem of this system. In order to use the feedback optimal control approach the lateral dynamics is expressed in a state vector form with adding the control forces to the equations of motion.

\section{MODELLING}

In this system, a pendulum with a torque $T(t)$, is attached to the side of cart by means of a pivot which allows the pendulum to swing in the $x y$-plane. A cart equipped with a motor exerts force $F(t)$, provides horizontal motion of the cart on a friction track, see Fig 1 . The purpose of the torque $T(t)$ and the force $F(t)$ is that keeping the pendulum balanced upright.

\section{A. Formation of the problem :}

By assuming that the pendulum is a thin rod with length $l$. Then, applying Newton's second law to the linear and angular displacement, the equations of motion are [4]

$$
\begin{gathered}
(M+m) \ddot{x}+\varepsilon \dot{x}+m \frac{l}{2} \ddot{\theta} \cos \theta-m \frac{l}{2} \dot{\theta}^{2} \sin \theta=F(t) \\
m \frac{l}{2} \cos \theta \ddot{x}+\frac{1}{3} m l^{2} \ddot{\theta}=T(t)+m g \frac{l}{2} \sin \theta
\end{gathered}
$$

where $M$ is the cart mass, $m$ is the pendulum mass, $x(t)$ is displacement of the center of mass of the cart from the center of the inertial frame, $\theta(t)$ is the angle between the pendulum and the top vertical.

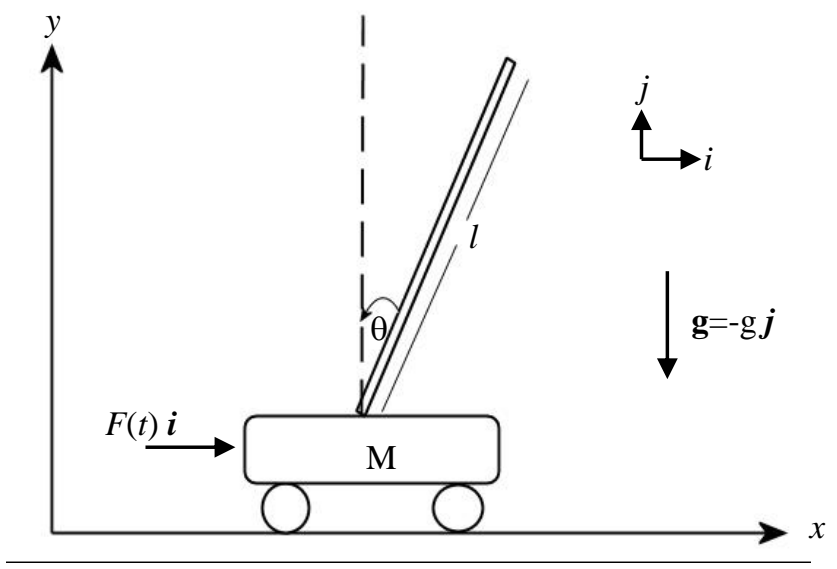

Fig. 1. Inverted Pendulum system

After some calculation, we can obtain

$$
\begin{aligned}
\ddot{x}= & \frac{4}{3 \alpha(\theta)} F(t)-\frac{2 \cos \theta}{l \alpha(\theta)} T(t)+\frac{2 m l \sin \theta}{3 \alpha(\theta)} \dot{\theta}^{2}- \\
& -\frac{4 \varepsilon}{3 \alpha(\theta)} \dot{x}-\frac{m g \sin 2 \theta}{2 \alpha(\theta)} \\
\ddot{\theta}= & \frac{4(M+m)}{m l^{2} \alpha(\theta)} T(t)-\frac{2 \cos \theta}{l \alpha(\theta)} F(t)-\frac{m \sin 2 \theta}{2 \alpha(\theta)} \dot{\theta}^{2}+ \\
& +\frac{2 \varepsilon \cos \theta}{l \alpha(\theta)} \dot{x}+\frac{2(M+m) g \sin \theta}{l \alpha(\theta)}
\end{aligned}
$$


where

$\alpha(\theta)=\frac{4}{3}(M+m)-m \cos ^{2} \theta=\frac{1}{3}(4 M+m)+m \sin ^{2} \theta$

and $g$ is the acceleration due to gravity and equal $9.8 \mathrm{~m} / \mathrm{s}$.

By introducing the following variables for a more convenient form of the equations (3) and (4)

$$
\dot{\boldsymbol{y}}=\boldsymbol{f}(\boldsymbol{y})=\left[\begin{array}{c}
y_{3} \\
y_{4} \\
\frac{1}{\alpha\left(y_{2}\right)}\left(\frac{4}{3} F(t)-\frac{2 \cos y_{2}}{l} T(t)-\frac{4}{3} \varepsilon y_{3}+\frac{2 m l \sin y_{2}}{3} y_{4}^{2}-\frac{m g \sin 2 y_{2}}{2}\right) \\
\frac{1}{\alpha\left(y_{2}\right)}\left(\frac{4(M+m)}{m l^{2}} T(t)-\frac{2 \cos y_{2}}{l} F(t)-\frac{m \sin 2 y_{2}}{2} y_{4}^{2}+\frac{2 \varepsilon \cos y_{2}}{l} y_{3}+\frac{2(M+m) g \sin y_{2}}{l}\right)
\end{array}\right]
$$

where

$$
\alpha\left(y_{2}\right)=\frac{1}{3}(4 M+m)+m \sin ^{2} y_{2}
$$

It is well known that for (7) with no control $(F(t)=0$ and $T(t)=0)$, the cart at rest with the pendulum in the upright position is an unstable equilibrium, while the cart at rest with the pendulum in the downward position is a stable equilibrium. Our concern is that when the pendulum at the unstable equilibrium point, so in the following section the system will be linearized about the unstable equilibrium $(0,0,0,0)^{T}$.

\section{B. Linearization:}

Now, by putting (7) in the following form

$$
\dot{\boldsymbol{y}}=C(\boldsymbol{y}) \boldsymbol{y}+D(\boldsymbol{y}) \boldsymbol{u}
$$

where

$$
C(\boldsymbol{y})=\left[\begin{array}{cccc}
0 & 0 & 1 & 0 \\
0 & 0 & 0 & 1 \\
0 & -\frac{m g}{\alpha\left(y_{2}\right)} \frac{\sin 2 y_{2}}{2 y_{2}} & -\frac{4 \varepsilon}{3 \alpha\left(y_{2}\right)} & \frac{2 m l \sin y_{2}}{3 \alpha\left(y_{2}\right)} y_{4} \\
0 & \frac{2(M+m) g}{l \alpha\left(y_{2}\right)} \frac{\sin y_{2}}{y_{2}} & \frac{2 \varepsilon \cos y_{2}}{l \alpha\left(y_{2}\right)} & -\frac{m \sin 2 y_{2}}{2 \alpha\left(y_{2}\right)} y_{4}
\end{array}\right]
$$

$$
D(\boldsymbol{y})=\left[\begin{array}{cc}
0 & 0 \\
0 & 0 \\
\frac{4}{3 \alpha\left(y_{2}\right)} & -\frac{2 \cos y_{2}}{l \alpha\left(y_{2}\right)} \\
-\frac{2 \cos y_{2}}{l \alpha\left(y_{2}\right)} & \frac{4(M+m)}{m l^{2} \alpha\left(y_{2}\right)}
\end{array}\right]
$$

and

$$
\boldsymbol{u}=\left[\begin{array}{ll}
F(t) & T(t)
\end{array}\right]^{T}
$$

We obtain the following equivalent first-order system
The system (9) will be linearized about the nominal trajectory $(0,0,0,0)^{T}$. Now by applying that $\lim _{y_{2} \rightarrow 0} \frac{\sin y_{2}}{y_{2}}=1$ and $\lim _{y_{2} \rightarrow 0} \cos y_{2}=1$, we can deduce that

$$
\dot{\boldsymbol{y}}=A \boldsymbol{y}+B \boldsymbol{u}
$$

where

$$
A=\left[\begin{array}{cccc}
0 & 0 & 1 & 0 \\
0 & 0 & 0 & 1 \\
0 & -\frac{3 m g}{4 M+m} & -\frac{4 \varepsilon}{4 M+m} & 0 \\
0 & \frac{6(M+m) g}{l(4 M+m)} & \frac{6 \varepsilon}{l(4 M+m)} & 0
\end{array}\right]
$$

and

$$
B=\left[\begin{array}{cc}
0 & 0 \\
0 & 0 \\
\frac{4}{4 M+m} & -\frac{6}{l(4 M+m)} \\
-\frac{6}{l(4 M+m)} & \frac{12(M+m)}{m l^{2}(4 M+m)}
\end{array}\right]
$$

This linearized system (13) allow to apply the feedback optimal control to balance the inverted pendulum around the point of linearization, as seeing in the following section.

\section{Closed LoOP (FEedBACK) OPTIMAL CONTROL PROBLEM}

The feedback optimal control problem is introduced to find optimal solutions minimize a certain performance index starting from a generic initial state $y_{0}$, The outcome is a control law written in terms of the time and the initial state, $v=v\left(y_{0}, t_{0}, t\right), t_{0} \leq t \leq t_{f}$. 
This represents a closed loop solution: given any initial state $y_{0}$ at the time $t_{0}$, it is possible to evaluate the optimal solution starting from such state up to the final target. If for any reason the state is perturbed and assumes a new value $y_{0}^{\prime}=y_{0}+\delta x, t_{0}^{\prime}=t_{0}+\delta t$, we are able to compute the new optimal solution by simply evaluating $v=v\left(y_{0}{ }_{0}, t_{0}, t\right)$, avoiding, in this way, the solution of another two-point boundary value problem. Thus, a trajectory designed in this way has the property to respond to errors that occur during the transfer. Another important aspect of this approach is the robustness of the solution. Once the optimal feedback control problem is solved, the solution $v=v\left(y_{0}, t_{0}, t\right)$ is available. Analyzing this function, the control law that is less sensitive to changes in the initial conditions can be chosen as nominal solution. This solution is said to be robust with respect to the initial conditions.

\section{A. Solving the Feedback Optimal Linear Quadratic Terminal} Controller Using the Generating Function Technique

Consider the problem of minimizing the following performance index , [7] , [8]

$$
J=\frac{1}{2} y_{f}^{T} Q_{f} y_{f}+\frac{1}{2} \int_{t_{0}}^{t_{f}}\left(y^{T} Q y+v^{T} R v\right) d t
$$

subject to the linear dynamics

$$
y=A y+B v
$$

and with the given initial and final conditions

$$
y\left(t_{0}\right)=y_{0} \quad \text { and } \quad y\left(t_{f}\right)=y_{f}
$$

According to the classical theory, the Hamiltonian of the optimal control problem is

$$
H=\frac{1}{2}\left(y^{T} Q y+v^{T} R v\right)+\lambda^{T}(A y+B v)
$$

where the set of Lagrangian multipliers has been introduced. From the optimality condition

$$
\frac{\partial H}{\partial v}=0
$$

It is possible to get an explicit expression for the control in terms of the Lagrangian multipliers

$$
v=-R^{-1} B^{T} \lambda
$$

Substituting the expression of given by equation (19), the Hamiltonian (17) turns out to be

$$
H=\frac{1}{2}\left(\begin{array}{l}
y \\
\lambda
\end{array}\right)^{T}\left[\begin{array}{cc}
Q & A^{T} \\
A & -B R^{-1} B^{T}
\end{array}\right]\left(\begin{array}{l}
y \\
\lambda
\end{array}\right)
$$

while the dynamics of the system and that of the Lagrange multipliers reduces to

$$
\left(\begin{array}{l}
\dot{y} \\
\dot{\lambda}
\end{array}\right)=\left[\begin{array}{cc}
A & -B R^{-1} B^{T} \\
-Q & -A^{T}
\end{array}\right]\left(\begin{array}{l}
y \\
\lambda
\end{array}\right)
$$

Suppose now that we have a generating function $F_{2}\left(y, \lambda_{0}, t, t_{0}\right)$ for the transformation between a fixed state $\left(y_{0}, \lambda_{0}, t_{0}\right)$ and a moving state $\left(y, \lambda, t_{0}\right)$. This transformation is canonical because it preserves the area in the phase space and in addition generates the identity transformation at $t=t_{0}$. we can derive this generating functions and their associated relations for this canonical transformation from HamiltonJacobi PDE

$$
\begin{gathered}
\lambda=\frac{\partial F_{2}\left(y, \lambda_{0}, t, t_{0}\right)}{\partial y} \\
y_{0}=\frac{\partial F_{2}\left(y, \lambda_{0}, t, t_{0}\right)}{\partial \lambda_{0}} \\
0=\frac{\partial F_{2}\left(y, \lambda_{0}, t, t_{0}\right)}{\partial t}+H\left(y, \frac{\partial F_{2}\left(y, \lambda_{0}, t, t_{0}\right)}{\partial y}, t\right)
\end{gathered}
$$

Since the Hamiltonian is quadratic, $F_{2}$ can be put in a quadratic form as follows

$$
F_{2}\left(y, \lambda_{0}, t, t_{0}\right)=\frac{1}{2}\left(\begin{array}{c}
y \\
\lambda_{0}
\end{array}\right)^{T}\left[\begin{array}{ll}
F_{y y}\left(t, t_{0}\right) & F_{y \lambda_{0}}\left(t, t_{0}\right) \\
F_{\lambda_{0} y}\left(t, t_{0}\right) & F_{\lambda_{0} \lambda_{0}}\left(t, t_{0}\right)
\end{array}\right]\left(\begin{array}{c}
y \\
\lambda_{0}
\end{array}\right)
$$

which can be used to find the unknown boundary conditions using the given ones. From the properties of $F_{2}$ we have

$$
\lambda=\frac{\partial F_{2}}{\partial y}=\left(\begin{array}{ll}
F_{y y} & F_{y \lambda_{0}}
\end{array}\right)\left(\begin{array}{c}
y \\
\lambda_{0}
\end{array}\right)
$$

The Hamiltonian (20) can be expressed as a function of $\left(y, \lambda_{0}\right)$ by using equation $(21)$

$$
H=\frac{1}{2}\left(\begin{array}{c}
y \\
\lambda_{0}
\end{array}\right)^{T}\left[\begin{array}{cc}
I & F_{y y} \\
0 & F_{\lambda_{0} y}
\end{array}\right]\left[\begin{array}{cc}
Q & A^{T} \\
A & -B R^{-1} B^{T}
\end{array}\right]\left[\begin{array}{cc}
I & 0 \\
F_{y y} & F_{y \lambda_{0}}
\end{array}\right]\left(\begin{array}{c}
y \\
\lambda_{0}
\end{array}\right)
$$

Since the Hamiltonian at the fixed state can be taken zero without any loss of generality, then the Hamiltonian of the moving state and the generating function satisfy the HamiltonJacobi PDE (22.c)

$$
\begin{aligned}
0= & \left(\begin{array}{c}
y \\
\lambda_{0}
\end{array}\right)^{T}\left\{\left[\begin{array}{ll}
\dot{F}_{y y} & \dot{F}_{y \lambda_{0}} \\
\dot{F}_{\lambda_{0} y} & \dot{F}_{\lambda_{0} \lambda_{0}}
\end{array}\right]+\right. \\
& \left.+\left[\begin{array}{cc}
I & F_{y y} \\
0 & F_{\lambda_{0} y}
\end{array}\right]\left[\begin{array}{cc}
Q & A^{T} \\
A & -B R^{-1} B^{T}
\end{array}\right]\left[\begin{array}{cc}
I & 0 \\
F_{y y} & F_{y \lambda_{0}}
\end{array}\right]\right\}\left(\begin{array}{c}
y \\
\lambda_{0}
\end{array}\right)
\end{aligned}
$$

whose sub-matrix components provide the following set of matrix ODEs for (Riccati Equations [2]) $F_{y y}\left(t, t_{0}\right)$,

$$
\begin{array}{r}
F_{y \lambda_{0}}\left(t, t_{0}\right)=F_{\lambda_{0} y}^{T}\left(t, t_{0}\right) \text {, and } F_{\lambda_{0} \lambda_{0}}\left(t, t_{0}\right) \\
\dot{F}_{y y}+Q+F_{y y} A+A^{T} F_{y y}-F_{y y} B R^{-1} B^{T} F_{y y}=0, \\
\dot{F}_{y \lambda_{0}}+A^{T} F_{y \lambda_{0}}-F_{y y} B R^{-1} B^{T} F_{y \lambda_{0}}=0,
\end{array}
$$




$$
\dot{F}_{\lambda_{0} \lambda_{0}}-F_{\lambda_{0} y} B R^{-1} B^{T} F_{y \lambda_{0}}=0,
$$

The initial conditions which verify the identity transformation at $t=t_{0}$ are

$$
\begin{aligned}
& F_{y y}\left(t_{0}, t_{0}\right)=0_{n \times n}, \\
& F_{y \lambda_{0}}\left(t_{0}, t_{0}\right)=I_{n \times n}, \\
& F_{\lambda_{0} \lambda_{0}}\left(t_{0}, t_{0}\right)=0_{n \times n}
\end{aligned}
$$

\section{B. Computing the Generating function}

\section{- The HCP}

We compute $F_{2}\left(y, \lambda_{0}, t, t_{f}\right)$ by using $t_{f}$ as our initial time, then we have from (23)

$$
y_{0}=\frac{\partial F_{2}\left(y, \lambda_{0}, t, t_{0}\right)}{\partial \lambda_{0}}=F_{\lambda_{0} y} y_{f}+F_{\lambda_{0} \lambda_{0}} \lambda_{0}
$$

Since we have $y_{0}$ and $y_{f}$ are given the initial Lagrange multiplier can be evaluated through

$$
\lambda_{0}=F_{\lambda_{0} \lambda_{0}}^{-1}\left(t_{f}, t_{0}\right)\left(y_{0}-F_{\lambda_{0} y}\left(t_{f}, t_{0}\right) y_{f}\right)
$$

Using (30) we can get the optimal trajectory by forward integration of (21). Since this relation (30) is valid for any initial time $t \leq t_{f}$, we have

$$
\lambda(t)=F_{\lambda_{0} \lambda_{0}}^{-1}\left(t_{f}, t\right)\left(y(t)-F_{\lambda_{0} y}\left(t_{f}, t\right) y_{f}\right)
$$

and therefore the control can be given by from (21)

$$
v(t)=-R^{-1} B^{T} F_{\lambda_{0} \lambda_{0}}^{-1}\left(t_{f}, t\right)\left(y(t)-F_{\lambda_{0} y}\left(t_{f}, t\right) y_{f}\right)
$$

- The SCP

We compute $F_{1}$ from Legendre transformation as follows

$$
F_{1}\left(y, y_{0}, t, t_{0}\right)=F_{2}\left(y, \lambda_{0}, t, t_{0}\right)-y_{0}^{T} \lambda_{0},
$$

Substituting $y_{0}$ from (29) we get after some algebraic manipulations

$$
F_{1}\left(y, y_{0}, t, t_{0}\right)=\frac{1}{2}\left(\begin{array}{c}
y \\
y_{0}
\end{array}\right)^{T}\left[\begin{array}{cc}
F_{y y}-F_{y \lambda_{0}} F_{\lambda_{0} \lambda_{0}}^{-1} F_{\lambda_{0} y} & F_{y \lambda_{0}} F_{\lambda_{0} \lambda_{0}}^{-1} \\
F_{\lambda_{0} \lambda_{0}}^{-1} F_{\lambda_{0} y} & -F_{\lambda_{0} \lambda_{0}}^{-1}
\end{array}\right]\left(\begin{array}{c}
y \\
y_{0}
\end{array}\right)
$$

then we have

$$
\begin{aligned}
& \lambda_{0}=F_{\lambda_{0} \lambda_{0}}^{-1}\left(t_{f}, t_{0}\right)\left(y_{0}-F_{\lambda_{0} y}\left(t_{f}, t_{0}\right) y_{f}\right), \\
& \lambda_{f}=F_{y \lambda_{0}} F_{\lambda_{0} \lambda_{0}}^{-1}\left(t_{f}, t_{0}\right) y_{0}+\left(F_{y y}-F_{y \lambda_{0}} F_{\lambda_{0} \lambda_{0}}^{-1} F_{\lambda_{0} y}\right)\left(t_{f}, t_{0}\right) y_{f}, \\
& \lambda_{f}=Q_{f} y_{f},
\end{aligned}
$$

By equating the second and the third equations we get a relation between $y_{0}$ and $y_{f}$ which can be plugged into the first equation to get

$$
\begin{aligned}
\lambda_{0}=\{ & \left\{F_{\lambda_{0} \lambda_{0}}^{-1}-\right. \\
& \left.\left.-F_{\lambda_{0} \lambda_{0}}^{-1} F_{\lambda_{0} y}\left(Q_{f}-F_{y y}+F_{y \lambda_{0}} F_{\lambda_{0} \lambda_{0}}^{-1} F_{\lambda_{0} y}\right)^{-1} F_{y \lambda_{0}} F_{\lambda_{0} \lambda_{0}}^{-1}\right]\left(t_{f}, t_{0}\right)\right\} y_{0},
\end{aligned}
$$

Using (32) we can get the optimal trajectory by forward integration of (21). Since this relation (32) is valid for any initial time $t \leq t_{f}$, we have

$$
\begin{aligned}
\lambda(t)= & \left\{\left[F_{\lambda_{0} \lambda_{0}}^{-1}-\right.\right. \\
& \left.\left.-F_{\lambda_{0} \lambda_{0}}^{-1} F_{\lambda_{0} y}\left(Q_{f}-F_{y y}+F_{y \lambda_{0}} F_{\lambda_{0} \lambda_{0}}^{-1} F_{\lambda_{0} y}\right)^{-1} F_{y \lambda_{0}} F_{\lambda_{0} \lambda_{0}}^{-1}\right]\left(t_{f}, t_{0}\right)\right\} y(t),
\end{aligned}
$$

and the control is given by

$$
\begin{aligned}
v(t)= & -R^{-1} B^{T}\left\{\left[F_{\lambda_{0} \lambda_{0}}^{-1}-\right.\right. \\
& \left.\left.-F_{\lambda_{0} \lambda_{0}}^{-1} F_{\lambda_{0} y}\left(Q_{f}-F_{y y}+F_{y \lambda_{0}} F_{\lambda_{0} \lambda_{0}}^{-1} F_{\lambda_{0} y}\right)^{-1} F_{y \lambda_{0}} F_{\lambda_{0} \lambda_{0}}^{-1}\right]\left(t_{f}, t_{0}\right)\right\} y(t),
\end{aligned}
$$

When $Q_{f}$ is large enough the SCP solution converges to the $\mathrm{HCP}$ one.

\section{NUMERICAL EXAMPLE}

The values of the parameters for our numerical example are given as follow,

$M=0.8 \mathrm{~kg} \quad, \quad m=0.21 \mathrm{~kg} \quad$ and $\quad \varepsilon=2.1$.

and

$$
\begin{aligned}
& t_{0}=0 \quad \text { and } \\
& y_{0}=\left(\begin{array}{lllll}
0.001 & 0.001 & 0.0002 & 0.0001
\end{array}\right)^{T}, \\
& t_{f}=0.45 \mathrm{~s} \quad \text { and } \quad \boldsymbol{y}_{f}=\left(\begin{array}{lllll}
0.0 & 0.0 & 0.0 & 0.0
\end{array}\right)^{T} .
\end{aligned}
$$

Then, by substituting in (14) and (15) we have

$$
\begin{aligned}
A & =\left[\begin{array}{cccc}
0 & 0 & 1 & 0 \\
0 & 0 & 0 & 1 \\
0 & -1.8106 & -2.4633 & 0 \\
0 & 28.5506 & 6.0574 & 0
\end{array}\right], \\
B & =\left[\begin{array}{cc}
0 & 0 \\
0 & 0 \\
1.1730 & -2.8845 \\
-2.8845 & 45.4851
\end{array}\right] .
\end{aligned}
$$

and by putting 


$$
\begin{aligned}
Q & =\left[\begin{array}{cccc}
100 & 0 & 0 & 0 \\
0 & 100 & 0 & 0 \\
0 & 0 & 1 & 0 \\
0 & 0 & 0 & 1
\end{array}\right], \\
R & =\left[\begin{array}{ll}
1 & 0 \\
0 & 1
\end{array}\right],
\end{aligned}
$$

and

$$
Q_{f}=\left[\begin{array}{llll}
1 & 0 & 0 & 0 \\
0 & 1 & 0 & 0 \\
0 & 0 & 1 & 0 \\
0 & 0 & 0 & 1
\end{array}\right] .
$$

We plot the resulting data in the following figures

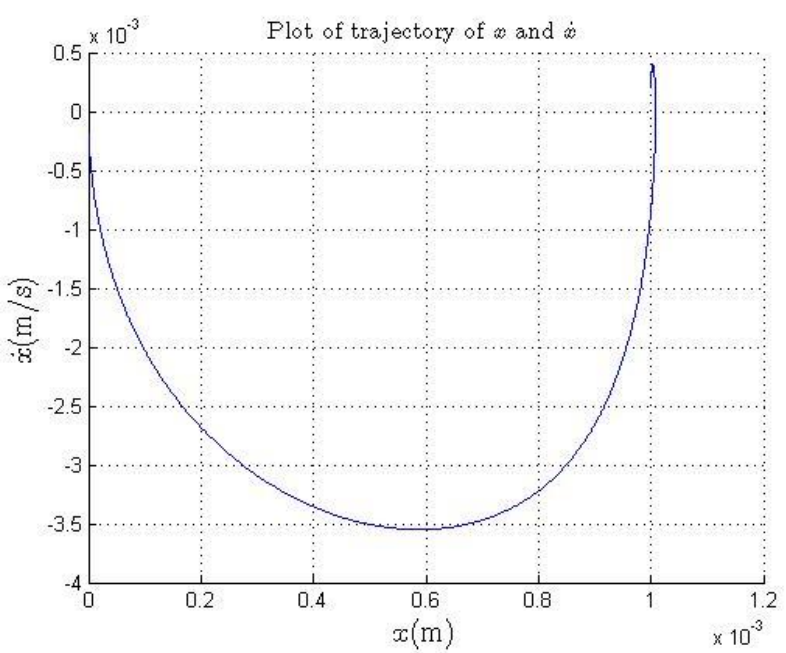

Fig. 2. Plot of trajectory of $\mathrm{x}$ and $\dot{\boldsymbol{x}}$

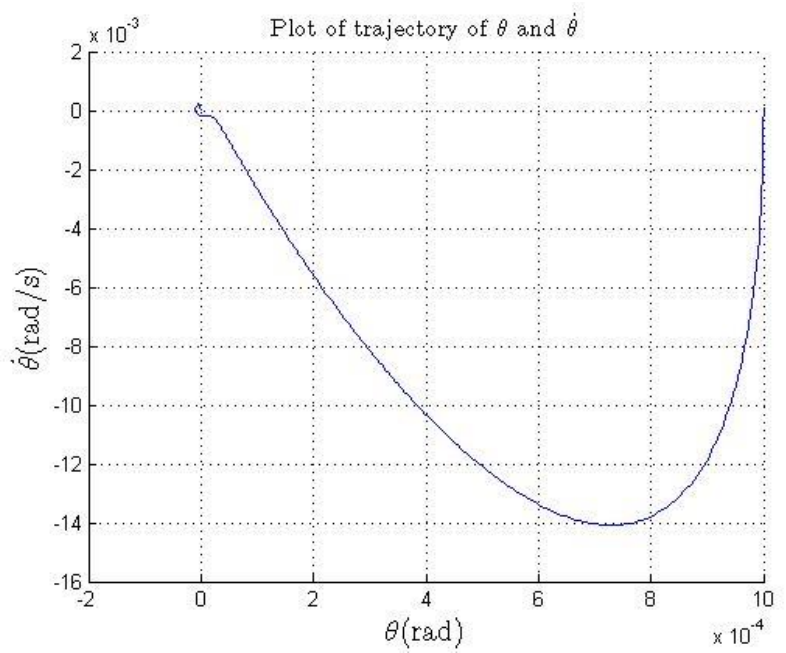

Fig. 3. Plot of trajectory of $\boldsymbol{\theta}$ and $\dot{\boldsymbol{\theta}}$

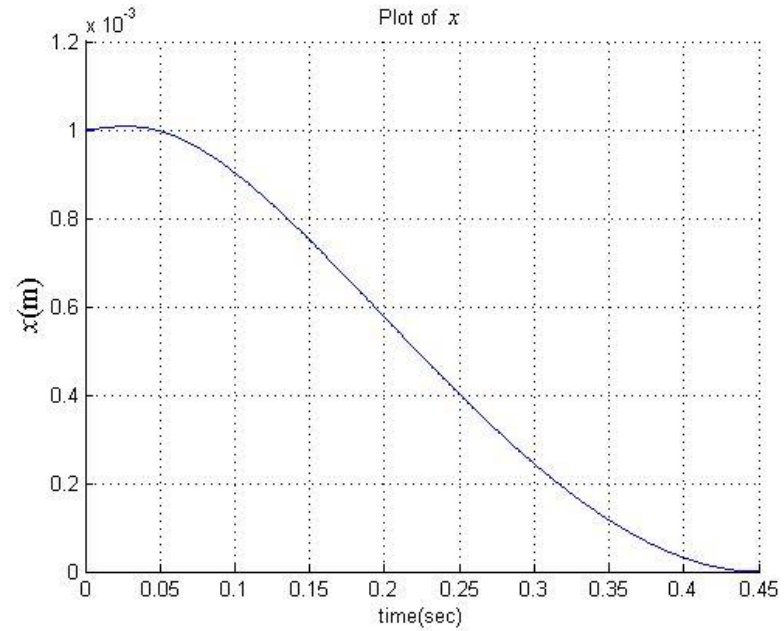

Fig. 4. Plot of $x(t)$

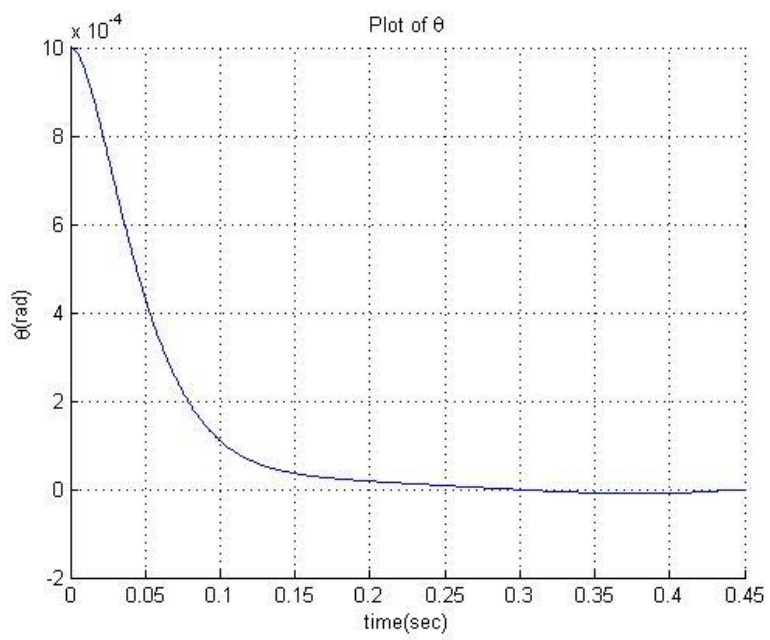

Fig. 5. Plot of $\theta(t)$

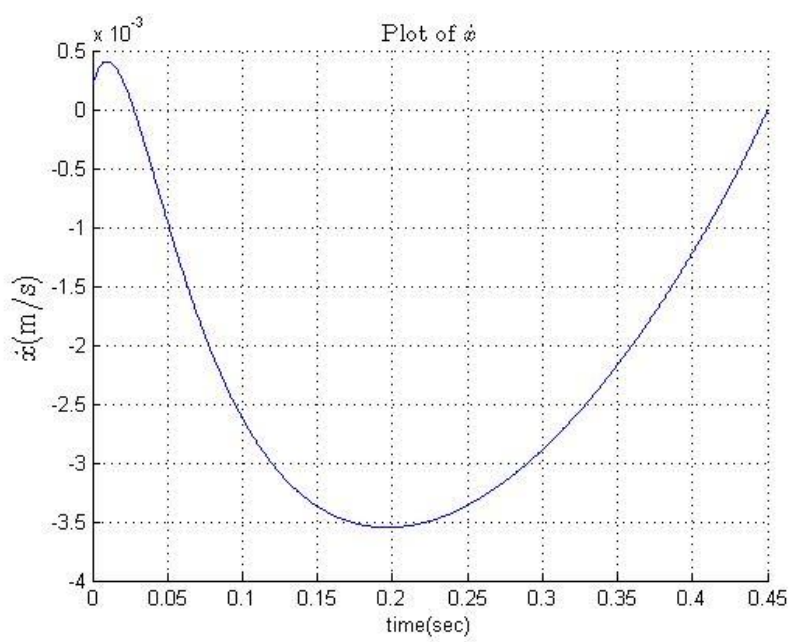

Fig. 6. Plot of $\dot{x}(t)$ 


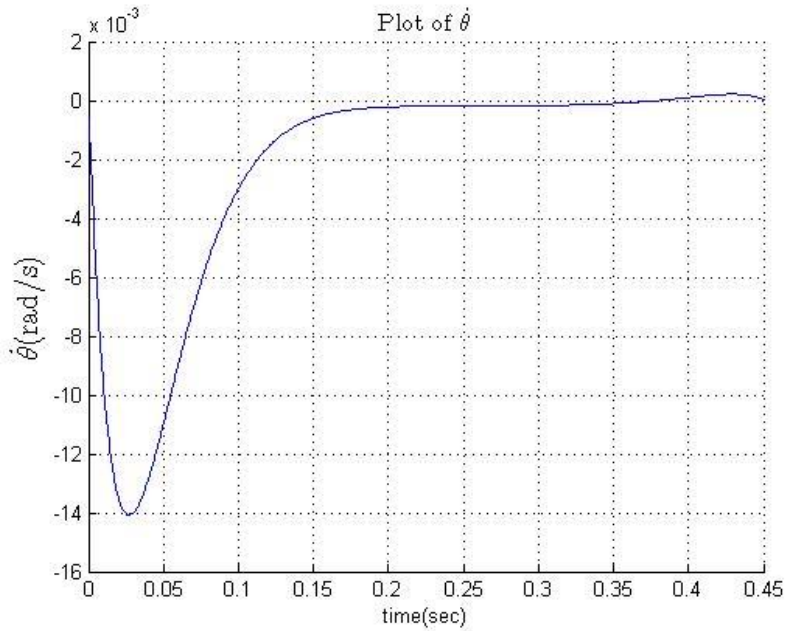

Fig. 7. Plot of $\dot{\theta}(t)$

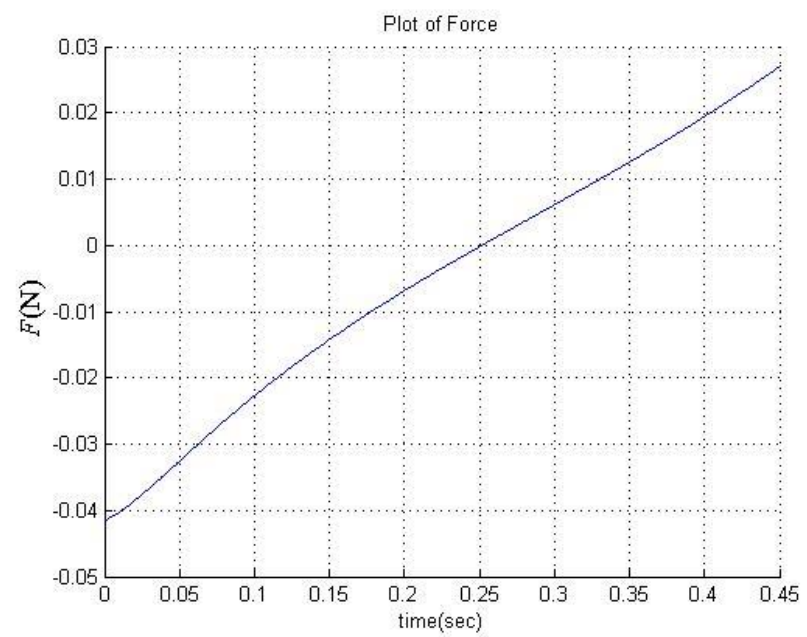

Fig. 8. Plot of $F(t)$

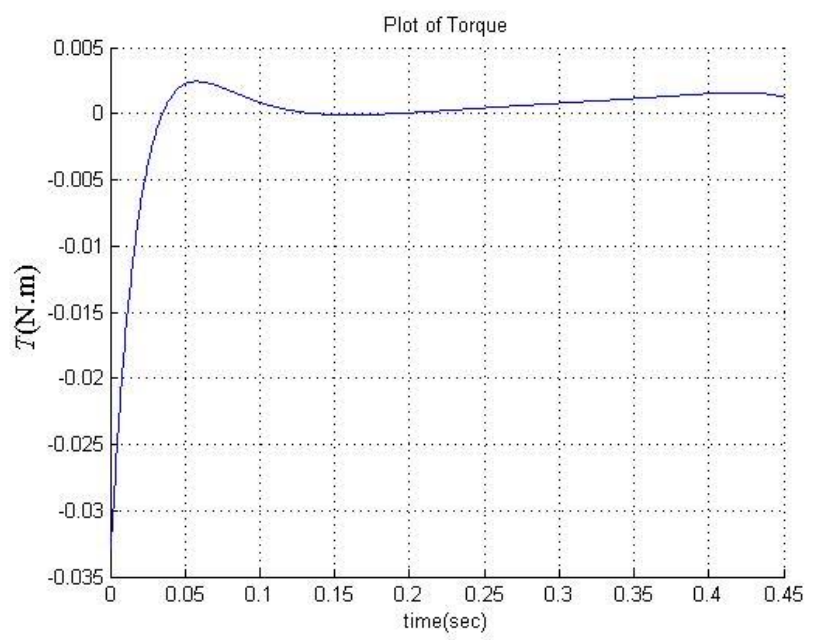

Fig. 9. Plot of $T(t)$

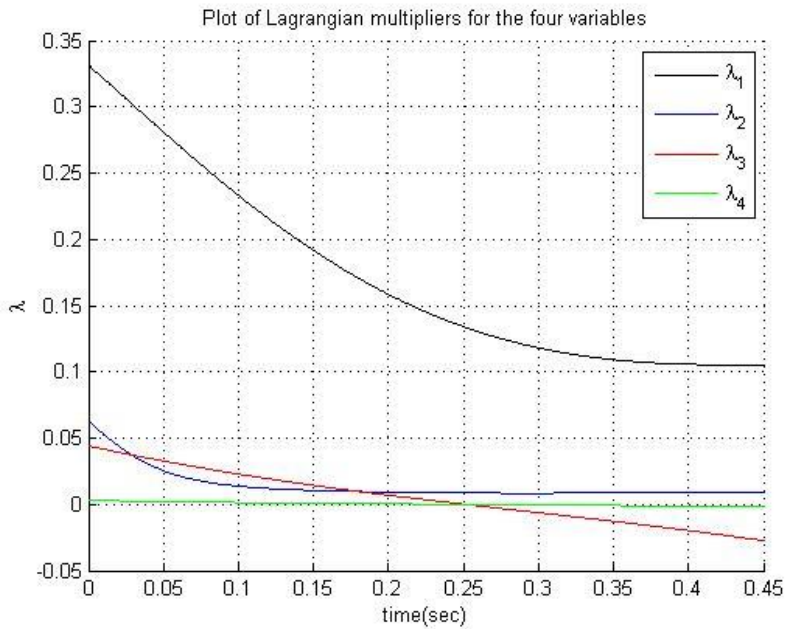

Fig. 10. Plot of Lagrangian multiliers

\section{CONCLUSION}

In the present study, the feedback optimal control of highly nonlinear inverted pendulum problem is solved by linearizing the original nonlinear dynamics. The linearized problem has been solved using the generating function technique where the method can be used for both hard and soft constraint boundary condition.

A proof exists in the very simple case of linear hard constraint problem (the final state is fully specified) and the figures (Fig2 to Fig 10) for the numerical example showed an excellent result in keeping the inverted pendulum in the unstable state for a short time. For a future work we can apply the same method feedback optimal control by using the generating function technique on the double inverted pendulum.

\section{REFERENCES}

[1] F. Atay, "Balancing the inverted pendulum using position feedback," Applied Mathematics Letter, vol. 12, pp. 51-56, 1999.

[2] A. E. Bryson and Y. Ho, Applied Optimal Control, London, England: Hemisphere Publishing Corp., 1975.

[3] K. Furuta, M. Yamakita and S. Kobayashi, "Swing-up control of inverted pendulum using pseudo-state feedback," Journal of Systems and Control Engineering, vol. 206, pp. 263-269, 1992.

[4] M. Landry, S. A. Campbell, K. Morris and C. O. Aguilar, "Dynamics of an Inverted Pendulum with Delayed Feedback Control," SIAM J. APPLIED DYNAMICAL SYSTEM, vol. 4, no. 2, pp. 333-351, 2005.

[5] R. Lozano and I. Fantoni, "Passivity based control of the inverted pendulums. In Normand-Cyrot," in Perspectives in control, 1998.

[6] H. Meier, Z. Farwig and H. Unbehauen, "Discrete computer control of a triple-inverted pendulum," Optical Control Applications and Methods,, vol. 11, pp. 157-171, 1990.

[7] C. Park and D. J. Scheeres, "Determination of optimal feedback terminal controllers for general boundary conditions using generating functions," Automatica, vol. 42, p. 869-875, 2006.

[8] C. Park and D. J. Scheeres, "Solutions of Optimal Feedback Control Problems with General Boundary Conditions using Hamiltonian Dynamics and Generating Functions," in Proceeding of the American Control Conference, Boston, Massachusetts, 2004.

[9] J. Sieber and B. Krauskopf, "Bifurcation analysis of an inverted pendulum with delayed feedback control near a triple-zero eigenvalue singularity," Nonlinearity, vol. 17, pp. 85-103, 2004. 